\title{
Discovery of a large-scale clumpy structure of the Lynx supercluster at $\mathrm{z} \sim 1.27$
}

\author{
Fumiaki Nakata ${ }^{1,2,3}$, Tadayuki Kodama ${ }^{2,3}$, Kazuhiro Shimasaku ${ }^{3}$, \\ Mamoru Doi ${ }^{4}$, Hisanori Furusawa ${ }^{5}$, Masaru Hamabe ${ }^{6}$, \\ Masahiko Kimura ${ }^{7}$, Yutaka Komiyama ${ }^{5}$, Satoshi Miyazaki ${ }^{5}$, \\ Sadanori Okamura ${ }^{3}$, Masami Ouchi ${ }^{3}$, Maki Sekiguchi ${ }^{8}$, \\ Masafumi Yagi ${ }^{2}$ and Naoki Yasuda ${ }^{8}$ \\ ${ }^{1}$ Department of Physics, University of Durham, South Road, Durham DH1 3LE, UK \\ email: fumiaki.nakata@durham.ac.uk \\ ${ }^{2}$ National Astronomical Observatory of Japan, Mitaka, Tokyo 181-8588, Japan \\ ${ }^{3}$ Department of Astronomy, University of Tokyo, Bunkyo-ku, Tokyo 113-0033, Japan \\ ${ }^{4}$ Institute of Astronomy, University of Tokyo, Mitaka, Tokyo 181-1500, Japan \\ ${ }^{5}$ Subaru Telescope, National Astronomical Observatory of Japan, Hilo, HI 96720, USA \\ ${ }^{6}$ Department of Mathematical and Physical Sciences, Faculty of Science, Japan Women's \\ University, Bunkyo-ku, Tokyo 112-8681, Japan \\ ${ }^{7}$ Department of Astronomy, Faculty of Science, Kyoto University, Sakyo-ku, \\ Kyoto 606-8502, Japan \\ ${ }^{8}$ Institute for Cosmic Ray Research, University of Tokyo, Kashiwa, Chiba 277-8582, Japan
}

\begin{abstract}
We report the discovery of a probable large-scale structure composed of many galaxy clumps around the known twin clusters at $z=1.26$ and $z=1.27$ in the Lynx region. Our analysis is based on deep, panoramic, and multi-colour imaging with the Suprime-Cam on the $8.2 \mathrm{~m}$ Subaru telescope. We apply a photometric redshift technique to extract plausible cluster members at $z \sim 1.27$ down to $\sim M^{*}+2.5$. From the 2-D distribution of these photometrically selected galaxies, we newly identify seven candidates of galaxy groups or clusters where the surface density of red galaxies is significantly high $(>5 \sigma)$, in addition to the two known clusters, comprising the largest most distant supercluster ever identified.
\end{abstract}

\section{Introduction}

Superclusters are the largest systems of galaxies, composed of multiple clusters of galaxies, extending over 10-20Mpc (e.g., Postman, Geller, Huchra 1988; Small et al. 1998). According to the N-body simulations which successfully reproduce the observed filamentary structures at the local Universe (e.g., Peacock et al. 2001), clusters of galaxies at $z \sim 1$ are still in the process of formation (e.g., Moore et al. 1998). Therefore, clusters of galaxies at this cosmological distance, especially superclusters, are important sites where we can directly see the process of structure formation and evolution and mass assembly to cluster cores.

The advent of the Suprime-Cam (Miyazaki et al. 2002), a huge format optical camera with a $30^{\prime}$ field of view on the prime focus of the $8.2 \mathrm{~m}$ Subaru telescope, has made it possible for us to view a $>10 \mathrm{Mpc}$ region all at once at high redshifts. By utilizing the great light-collection power of the Subaru telescope, we now target the most distant supercluster ever firmly identified with spectroscopy: i.e., the Lynx supercluster region at $z \sim 1.27$ composed of two known clusters, RXJ 0848.9+4452 at $z=1.26$ and RXJ 0848.6+4453 at $z=1.27$ (Stanford et al. 1997; Rosati et al. 1999). Throughout this article, we use the 
AB magnitude system. The adopted cosmological parameters are $\Omega_{0}=0.3$ and $\lambda_{0}=0.7$, which gives a physical scale of $11.7 h_{50}^{-1} \mathrm{kpc} \operatorname{arcsec}^{-1}$ at the cluster redshift.

\section{Observations, reduction and analysis}

The Lynx field was observed in November 2000-March 2001 with the Suprime-Cam. Total exposure times of $96,90,60$, and 54 mins were obtained in $V R i^{\prime} z^{\prime}$ respectively. The images are processed in a standard manner with IRAF and purpose-written software developed by us (Yagi et al. 2002). We register the $V, R$, and $i^{\prime}$ images with the $z^{\prime}$ image, and match their PSFs to a fixed value, $1^{\prime \prime}$ (FWHM). Taking an overlapped region for all the images in the four bands, the final area used in our analysis is restricted in a $26^{\prime} .4 \times 24^{\prime} .1$ region, which corresponds to $18.1 \times 17.5 h_{50}^{-2} \mathrm{Mpc}^{2}$ at $z=1.27$. We then constructed $i^{\prime}$-band selected sample using SExtractor v.2.2.0 (Bertin, Arnouts 1996). After excluding 411 stellar-like objects, a total of 35143 objects, whose magnitudes are brighter than $i^{\prime}=26.15(5 \sigma)$ in the $\sim 590 \mathrm{arcmin}^{2}$ area, are contained in the final catalog.

Since spectroscopic measurements for the $\sim 35,000$ galaxies are not practical, we exploit photo- $z$ technique as an observationally efficient method to largely subtract the foreground/background populations. We input the $V R i^{\prime} z^{\prime}$ magnitudes to the HYPERZ code (Bolzonella, Miralles \& Pelló 2000) to get estimated redshifts for all the individual galaxies in our $i^{\prime}$-band selected sample. By using the best estimate of photometric redshift of individual galaxies, we select the only galaxies within the range of $1 \leqslant z_{\text {phot }} \leqslant 1.35$ to isolate the plausible members associated to the supercluster at $z \sim 1.27$. The number of these photometrically selected candidates for cluster members (hereafter photo- $z$ selected candidates) is 2229 .

\section{Large-scale clumpy structure}

Figure 1 shows the spatial distribution on the sky of the photo- $z$ selected candidates at $z \sim 1.27$. The large and small dots indicate red and blue galaxies, redder and bluer respectively than a colour of the local Sab galaxies. The numbers of the red and blue galaxies are 575 and 1654, respectively. The contours indicate the local surface density of the 'red' photo- $z$ selected candidates calculated from the 10 nearest neighbors, corresponding to 2, 3, 4 and $5 \sigma$. Here $\sigma$ corresponds to the scatter (standard deviation) of the local surface density in the case of the random distribution of 575 galaxies over the $590 \mathrm{arcmin}^{2}$ field of view, i.e., $0.97 \mathrm{arcmin}^{-2}$. We do not include the blue galaxies here for the following reasons: (1) The accuracy of photo- $z$ is relatively poor for the blue galaxies because of their intrinsically weak $4000 \AA$ feature. (2) The red galaxies trace clusters or groups of galaxies more neatly than the blue galaxies due to the morphology (colour)-density relation (Dressler 1980, Dressler et al. 1997).

In Figure 1, we mark by large circles the areas where the local surface densities of the red galaxies are higher than $5 \sigma$ above the mean density. The cl1 and cl2 indicate the 2 known high redshift clusters at $z \sim 1.27$. We newly find 7 candidates of galaxy clusters or groups, where the local surface density is as large as those of the known clusters (gr1-gr7).

Figure 2 shows the $i^{\prime}-z^{\prime}$ versus $z^{\prime}$ diagram for each cluster/group candidate including the two known clusters. Galaxies within a circle of $1 h_{50}^{-1} \mathrm{Mpc}$ radius are plotted. The filled circles indicate the photo- $z$ selected candidates and the small dots indicate the other galaxies. For comparison, we also show the galaxies randomly selected from the low density $(<0 \sigma)$ regions in the bottom right panel of Figure 2. The dashed line in each panel shows a predicted colour-magnitude relation at $z=1.27$ for passively evolving galaxies formed at $z_{\mathrm{F}}=4.5$ (Kodama \& Arimoto 1997). Not only in the two known 


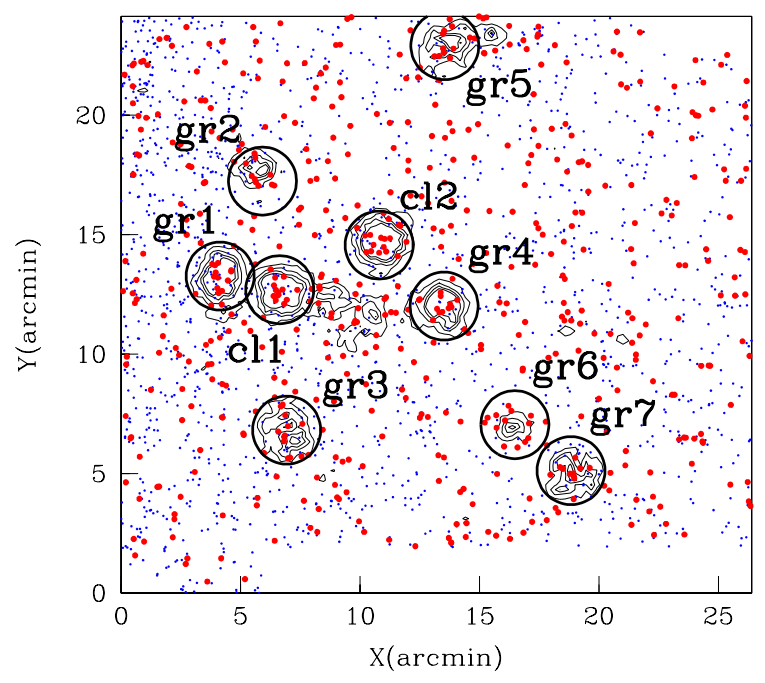

Figure 1. The 2-D distribution of photo- $z$ selected candidates on the sky. North is up, and east is to the left. The large and small dots indicate red and blue galaxies respectively. The contour levels are $2,3,4$ and $5 \sigma$. See the text for details. The radii of the large circles are $1 h_{50}^{-1} \mathrm{Mpc}$ at $z=1.27$.

clusters (cl1 and cl2) but also in our new candidates (gr1-gr7), can we find many red galaxies consistent with passive evolution which comprise the red sequences in these diagrams.

We also show the radial profile of each cluster and cluster/group candidate in Figure 3. Only those galaxies brighter than $i^{\prime}=26$ are counted because of increasing incompleteness toward fainter magnitudes. Here we subtract the remaining field contamination using the Subaru Deep Field data (e.g., Kashikawa et al. 2003; Ouchi et al. 2003) from each bin after normalizing counts of field galaxies with referring areas. The averaged surface number densities of all galaxies and the red galaxies are $4.0 \pm 3.6$ and $0.20 \pm 0.33 h_{50}^{2} \mathrm{Mpc}^{-2}$, respectively, to the same magnitude limit of $i^{\prime}=26$. We see a pronounced increase in galaxy density towards the center of most of our cluster/group candidates. The only exception is gr6 that shows no excess of galaxy density at the center. This may be due to the misidentification of the center of the group, or intrinsically non-axisymmetric structure. We combine the profiles of known clusters and those of our new candidates (groups) separately, and find that both of these combined profiles can be fitted very well with the King's law profile, $N(r)=N_{0}\left(1+r^{2} / R_{c}^{2}\right)^{-1}$ (King 1966, 1972), with $R_{\mathrm{c}}=0.23 \pm 0.03 h_{50}^{-1} \mathrm{Mpc}$ (cluster) and $R_{\mathrm{c}}=0.32 \pm 0.04 h_{50}^{-1} \mathrm{Mpc}$ (group), which is consistent with estimations for local clusters (Bahcall 1975).

We estimate the richness of each cluster/group candidate, using the $N_{0.5}$ indicator introduced by Hill \& Lilly (1991). This indicates the number of galaxies within a $0.5 \mathrm{Mpc}$ radius from the cluster center and within the magnitude range between $m_{1}$ and $m_{1}+3$ measured in the $R$ band at $z \sim 0.5$. Since the $R$ band at $z \sim 0.5$ roughly corresponds to the $z^{\prime}$-band at $z \sim 1.27$, we can make a direct comparison with their measurements. The cluster richness estimated based on $N_{0.5}$ is 1-2 for cl1 and cl2, while 0-1 for gr1-gr7 (See Table 4 of Hill \& Lilly 1991). Therefore we find that the candidates we newly find are poorer systems than the two known clusters. This is also suggested by the deep $(190 \mathrm{ks}$ 

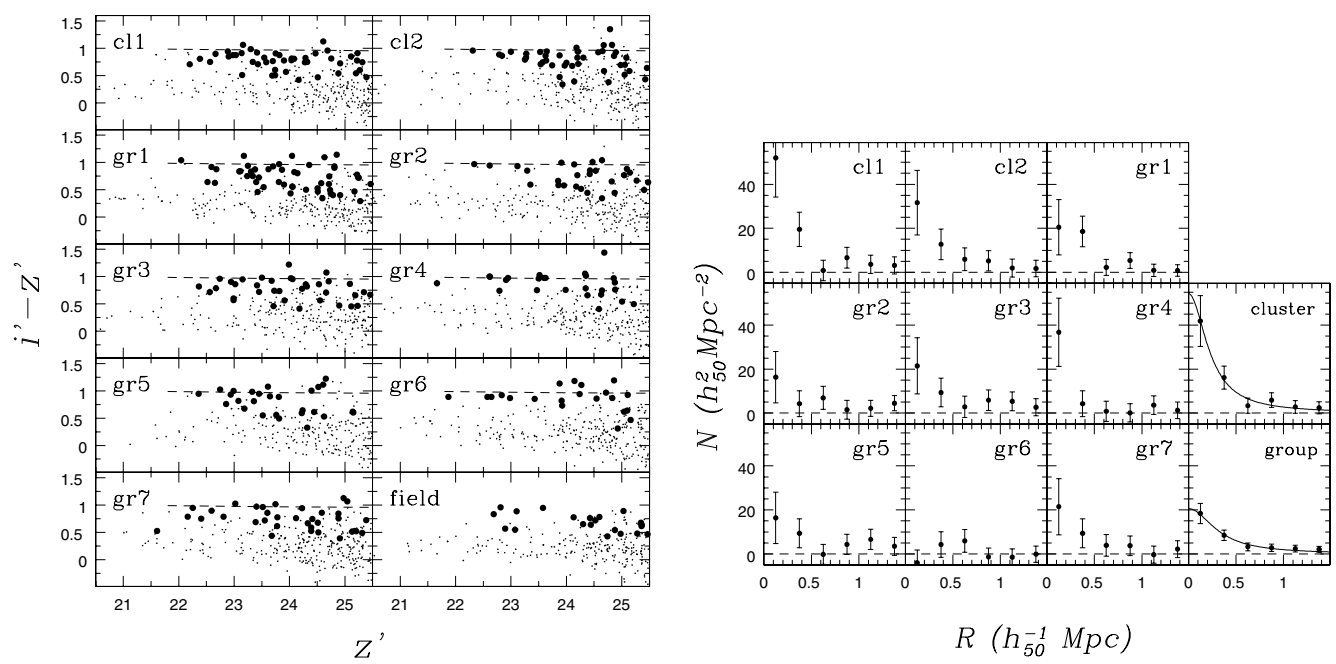

Figure 2 (left) : The $i^{\prime}-z^{\prime}$ versus $z^{\prime}$ diagrams of the galaxies in the known clusters (cl1 and cl2) and our new cluster/group candidates (gr1-gr7). See the text for details.

Figure 3 (right) : The surface number density of galaxies as a function of the distance from the cluster center. Cluster and group indicate the combined profiles of known clusters and those of our new candidates, respectively, and the best-fitted King's law profiles are also plotted by the solid lines.

exposure) X-ray data around the Lynx field taken by Chandra (Stanford et al. 2001; Stern et al. 2002). We compare the structures found in our optical data with those in the X-ray data. We cannot identify any counterparts (extended sources) in our cluster/group candidates except for the two known clusters. This may be because these candidates do not have dense or hot enough plasma to be detected in the Chandra data, whose limiting flux is $\approx 1.7 \times 10^{-16} \mathrm{ergs} \mathrm{cm}^{-2} \mathrm{~s}^{-1}$ in the $0.5-2 \mathrm{keV}$ band. We argue therefore that our 'Suprime-Cam imaging + photo- $z$ ' method is a very powerful technique in finding clusters or groups in the distant Universe even beyond $z>1$ out to $z \sim 1.3$. We note that this is practically the highest redshift structures of 'normal' galaxies that can be traced by optical images with our scheme (bracketing the $4000 \AA$-break region in the rest frame).

\section{Summary}

We have presented deep panoramic imaging of the Lynx supercluster field at $z \sim$ 1.27 taken with the Suprime-Cam on Subaru telescope. Our multicolor image covers a $26^{\prime} .4 \times 24^{\prime} .1\left(18.1 \times 17.5 h_{50}^{-1} \mathrm{Mpc}\right)$, allowing us for the first time to investigate the largescale structures spreading around the known cluster regions at this high redshift. By applying photo- $z$ technique, we have mapped out the spatial distributions of galaxies at $z \sim 1.27$ down to $\sim M^{*}+2.5$.

With this technique, we have newly discovered seven cluster/group candidates. These candidates show red color-magnitude sequences and centrally concentrated profiles, similar to those of the two known clusters in this field, indicating that they are likely to be real clusters/groups of galaxies at $z \sim 1.27$, comprising a large-scale supercluster over $\sim 18 h_{50}^{-1} \mathrm{Mpc}$ scale. The clumpiness of the structures suggests that its dynamical stage is young and they are in the process of assembly to a massive cluster. 


\section{References}

Bahcall, N. A. 1975 ApJ, 198, 249

Bertin, E., Arnouts, S. 1996 A\&AS, 177, 393

Bolzonella, M., Miralles, J.-M., Pelló, R. 2000 A\&A, 363, 476

Dressler, A. 1980 ApJ, 236, 351

Dressler, A. et al. 1997 ApJ, 490, 577

Hill, G. J. Lilly, S. J. 1991 ApJ, 367, 1

Kashikawa, N. et al. 2003 AJ, 125, 53

King, I. R. 1966 AJ, 71, 1

King, I. R. 1972 ApJ, 174, L123

Kodama, T., Arimoto, N. 1997 A\&A, 320, 41

Miyazaki, S. et al. 2002 PASJ, 54, 833

Moore, B., Governato, F., Quinn, T., Stadel, J., Lake, G. 1998 ApJ, 499, L5

Ouchi, M. et al. 2003 ApJ, 582, 60

Peacock, J. A. et al. 2001 Nature, 410, 169

Postman, M., Geller, M. J., Huchra, J. P. 1998 AJ, 95, 267

Rosati, P., Stanford, S. A., Eisenhardt, P. R., Elston, R., Spinrad, H., Stern, D., Dey, A. 1999 AJ, 118, 76

Small, T. A., Chung-Pei, M., Sargent, W. L. W., Hamilton, D. 1998 ApJ, 492, 45

Stanford, S. A., Elston, R., Eisenhardt, P. R., Spinrad, H., Stern, D., Dey A. 1997 AJ, 114, 2232

Stanford, S. A., Holden, B., Rosati, P., Tozzi, P., Borgani, S., Eisenhardt, P. R., Spinrad, H. 2001 ApJ, 552, 504

Stern, D. et al. 2002 AJ, 123, 2223

Yagi, M., Kashikawa, N., Sekiguchi, M., Doi, M., Yasuda, N., Shimasaku, K., Okamura, S. 2002 AJ, 123, 66 